\title{
Crystal structure, complex phase diagram and re-entrant phase transition in $\mathrm{NaSrPO}_{4}$
}

\author{
G. Nénert \\ Malvern Panalytical, Lelyweg 1, 7602 EA, Almelo, The Netherlands \\ gwilherm.nenert@malvernpanalytical.com
}

The crystal chemistry of $\mathrm{A}^{\mathrm{I}} \mathrm{B}^{\mathrm{II}} \mathrm{XO}_{4}\left(\mathrm{~A}^{\mathrm{I}}=\right.$ Alkali ion, $\mathrm{B}^{\mathrm{II}}=$ alkali-earth ion, $\left.\mathrm{X}=\mathrm{P}, \mathrm{V}, \mathrm{As}\right)$ is very rich and has been widely investigated, particularly the phosphate family [1]. In recent years, we have been investigated the crystal structures [2,3] and magnetic properties of some compositions within the $\mathrm{A}^{\mathrm{I}} \mathrm{B}^{\mathrm{II}} \mathrm{XO}_{4}$ series [4]. Besides the pure interest from a crystal chemistry point of view, the research activity related to this series of materials is driven mainly due to their ferroelectric, ferroelastic properties and possible applications as phosphors for LEDs $[1,5]$. Within the rich $\mathrm{A}^{\mathrm{I}} \mathrm{B}^{\mathrm{II}} \mathrm{VO}_{4}$ sub-family $(\mathrm{X}=\mathrm{V})$, we have recently found a new structural type: the larnite structure with the composition $\mathrm{NaSrVO}_{4}$ [3]. In this contribution, we are investigating its counter phosphate composition.

Despite its simple chemistry $\mathrm{NaSrPO}_{4}$ has never been reported so far. Here, we present the synthesis, crystal structure and phase transitions of this phosphate. Surprisingly, this material exhibits a complex structure ( 31 atoms in the asymmetric unit-cell, $Z=10)$ at room temperature characterized by a strongly under bonded $\mathrm{Na}$ atom. This under-bonded atom is responsible for the complex and rich phase diagram as function of temperature as illustrated in Fig. 1. $\mathrm{NaSrPO}_{4}$ exhibits 4 phase transitions between room temperature and $750^{\circ} \mathrm{C}$. Besides its rich phase diagram, $\mathrm{NaSrPO}_{4}$ exhibits a re-entrant phase transition slightly below $600^{\circ} \mathrm{C}$ before to reach a hexagonal paraelastic phase at high temperature. In addition, we show that the sequence of phase transitions is strongly driven by the history of the sample and several phases can be quenched at room temperature. Finally, the co-existence of Na channels within the structure with weakly bounded $\mathrm{Na}$ atoms makes this material a likely candidate for ionic conductivity.
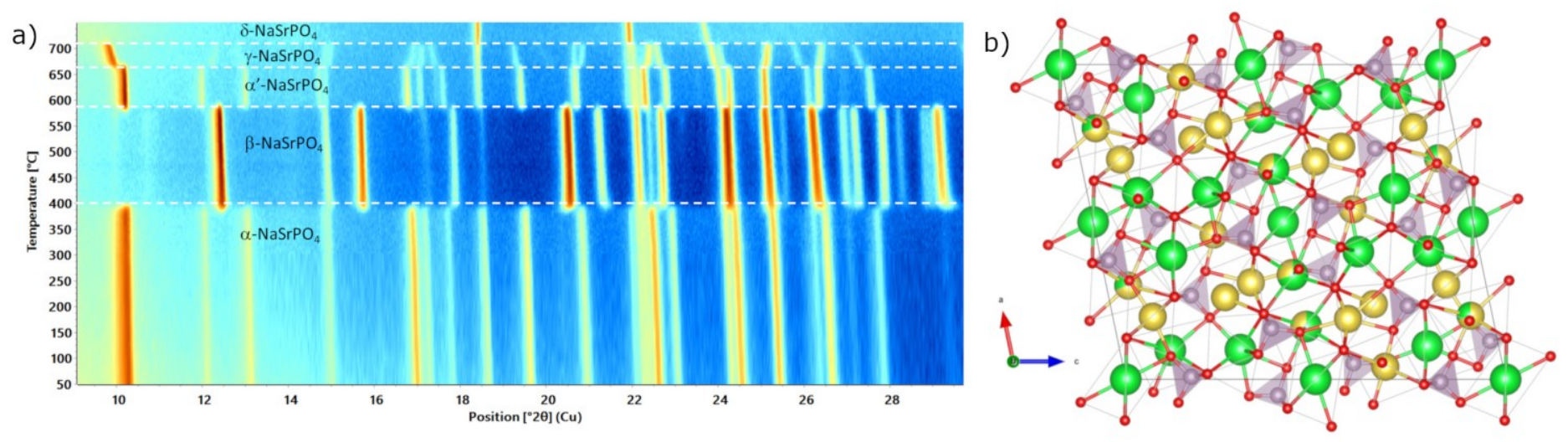

Figure 1. a) Temperature phase diagram of $\mathrm{NaSrPO}_{4}$ and b) crystal structure of the $\alpha$ re-entrant polymorph.

[1] Isupov, V. A., (2002). Ferroelectrics 274, 203.

[2] Nénert, G., O’Meara, P. , Degen, T. (2017). Phys. Chem. Minerals 44, 455.

[3] Nénert, G., (2017). Z. Kristallogr. 232, 669.

[4] Nénert, G., et al. (2013). Inorg. Chem. 52, 9627.

[5] Choi, S., Yun, Y. J. , Kim, S. J., Jung, H.-K. (2013) Opt. Lett. 38, 1346.

Keywords: phosphate; re-entrant phase transition; ab-initio structure solution; complex crystal structure 